\title{
ATLAS Score Clinical Classification
}

National Cancer Institute

\section{Source}

National Cancer Institute. AT LAS Score Clinical Classification. NCI Thesaurus. Code C147589.

A standardized scoring system created by Miller et al. in 2013 that assesses age, treatment with systemic antibiotics, leukocyte count, albumin and serum creatinine to predict treatment response or mortality from Clostridium difficile infection. 